\title{
PAGOS POR DESEMPEÑO PARA MEJORAR EL ESTADO NUTRICIONAL INFANTIL: IMPACTO DE LOS CONVENIOS DE APOYO PRESUPUESTARIO EN TRES REGIONES PERUANAS CON ALTA PREVALENCIA DE DESNUTRICIÓN CRÓNICA INFANTIL, 2010-2014
}

\author{
Viviana Cruzado de la Vega ${ }^{1,2, a}$
}

\begin{abstract}
RESUMEN
Objetivos. Estimar el impacto de un esquema de pago por desempeño, denominado convenios de apoyo presupuestario, aplicado por el Gobierno a las tres regiones con mayores tasas de desnutrición crónica infantil (DCl) en 2008, Apurimac, Ayacucho y Huancavelica, sobre indicadores de cobertura de servicios de cuidado infantil (vacunación, controles de crecimiento y desarrollo infantil, suplemento de hierro) y del estado nutricional del niño (desnutrición, anemia, diarrea). Mediante estos convenios se transferían recursos a los presupuestos de dichas regiones condicionados al cumplimiento de compromisos de gestión y metas de cobertura con el objetivo de mejorar el estado nutricional infantil. Materiales y métodos. A partir de los datos de la Encuesta Demográfica y de Salud Familiar de 2008 a 2014, se compara la evolución en los indicadores evaluados de una muestra de niños que residen en los ámbitos donde se suscribieron los convenios y una muestra de control, mientras los convenios estuvieron vigentes y en los años posteriores para reportar el estimador de diferencias en diferencias del impacto promedio de los convenios Resultados. se encuentran impactos positivos sobre el incremento de coberturas de vacunas del esquema básico y de la vacuna rotavirus y, a través de ellos, en la reducción de la ocurrencia de diarrea y desnutrición. Conclusiones. el esquema habría sido efectivo en activar la cadena mayor cobertura de vacunas y menor $\mathrm{DCl}$, pero no parece mejorar la cobertura de otras prestaciones como las atenciones de crecimiento y desarrollo del niño y entrega de suplementos de hierro al niño y gestante.
\end{abstract}

Palabras clave: Evaluación del impacto en la salud; Evaluación de programas y proyectos de salud; Reembolso de incentivo; Salud del niño; Transtornos de la nutrición del niño (fuente: DeCS BIREME).

\section{PAYMENT BY PERFORMANCE TO IMPROVE THE NUTRITIONAL STATUS OF CHILDREN: IMPACT OF BUDGET SUPPORT AGREEMENTS IN THREE PERUVIAN REGIONS WITH A HIGH PREVALENCE OF CHRONIC MALNUTRITION IN CHILDREN IN 2010-2014}

\begin{abstract}
Objectives. To estimate the impact of a payment scheme by performance, known as a budget support agreement, applied by the government in three regions in Peru with the highest rates of chronic malnutrition (CM) in children in 2008-Apurimac, Ayacucho, and Huancavelica-on indicators of health service coverage (immunization, childhood growth and development, and iron supplementation) and the nutritional status of children (malnutrition, anemia, and diarrhea). These agreements were used to transfer resources to the budgets of these regions with the condition of fulfilling management commitments and coverage goals with a view toward improving the nutritional status of children. Materials and methods. Based on data from the Demographic and Family Health Survey conducted from 2008 to 2014 evolution of the indicators in a sample of children residing in the areas where the support programs were signed was compared to that of a control sample in the period in which the agreements were in force and in the subsequent years to estimate differences in the impact of this support strategy. Results. There was a positive impact of the programs on the increase in vaccination coverage provided by the basic health system and rotavirus vaccination, which consequently reduced the rates of diarrhea and malnutrition. Conclusions. The scheme was effective in increasing the vaccination coverage and reducing CM but did not seem to improve the coverage of other benefits, including childhood growth and iron supplementation to children and mothers.
\end{abstract}

Key words: Health impact assessment; Program evaluation; Reimbursement, incentive; Child health; Child nutrition disorders (source: MeSH NLM).

\footnotetext{
Dirección de Calidad del Gasto Público, Dirección General de Presupuesto Público, Ministerio de Economía y Finanzas. Lima, Perú. Pontificia Universidad Católica del Perú. Lima, Perú.

Economista, magister en Economía Aplicada.

Este artículo está basado en el informe profesional realizado por la autora para optar el título de Licenciada en Economía de la Pontificia Universidad Católica del Perú.

Recibido: 04/07/2017 Aprobado: 20/09/2017 En línea: 29/09/2017
}

Citar como: Cruzado de la Vega V. Pagos por desempeño para mejorar estado nutricional infantil: impacto de los convenios de apoyo presupuestario en tres regiones peruanas con alta prevalencia de desnutrición cronica infantil, 2010-2014. Rev Peru Med Exp Salud Publica. 2017;34(3):365-76. doi: 10.17843/rpmesp.2017.343.2987 


\section{INTRODUCCIÓN}

A través de los pagos por desempeño se transfieren recursos condicionados al cumplimiento de metas o logros de algún resultado medible y previamente identificado entre dos partes. Su uso en las relaciones laborales se sustenta en que existe una asimetría de información en algunos contextos donde las acciones del trabajador (agente) no son observables por el empleador (principal), lo que origina comportamientos oportunistas.

Eldridge y Palmer (1) sostienen que los convenios que pagan de acuerdo al desempeño están diseñados para alinear los objetivos del agente a los del principal mediante un contrato que incentive al agente a esforzarse más. Sus ventajas, según Makinson (2): incrementa la productividad, clarifica los objetivos y mejora el involucramiento con las metas de la organización. Sin embargo, podrían desvirtuar comportamientos vinculados a motivaciones intrínsecas del agente con un interés afín al principal ${ }^{(3)}$.

Holmstrom y Milgron (4) mencionan las dificultades de su aplicación en el sector público debido a los múltiples objetivos de las entidades públicas, los que, también, son difíciles de medir; además, operan en un contexto político y una estructura jerárquica donde un trabajador o unidad debe rendir cuentas a varias unidades de mayor nivel jerárquico ${ }^{(5)}$. A lo dicho por estos autores, habría que añadir que cuando los pagos priorizan algunos resultados medibles frente a otros no observables, podría incentivar a los trabajadores a realizar prácticas no deseadas (por ejemplo: desmedro de calidad en la prestación de servicios por maximizar cantidad).

A pesar de estos desafíos, los mecanismos de pago por desempeño han sido empleados por varios gobiernos en diversos sectores, como Educación (6-10) y Salud ${ }^{(11-13)}$. Según Miller y Singer ${ }^{(14)}$, su aplicación a los sistemas de salud a los países de ingreso medio y bajo se ha extendido durante los últimos quince años. En América Latina, Argentina ${ }^{(12)}$ y Nicaragua ${ }^{(15)}$ han implementado esquemas para mejorar la calidad y acceso de servicios de salud materno infantil, y a nivel mundial, en Ruanda ${ }^{(13)}$, China ${ }^{(16)}$ India ${ }^{(17)}$, entre otros.

Una de las primeras experiencias de su aplicación fue emprendida en el 2009 por el Gobierno peruano, a través del Ministerio de Economía y Finanzas (MEF), mediante el Convenio de Apoyo Presupuestario (CAP), acuerdo establecido entre el Gobierno nacional y los gobiernos regionales (GR) con la finalidad de impulsar el logro de resultados de la política nacional para reducir la desnutrición crónica infantil $(\mathrm{DCl})$, denominado Programa Articulado Nutricional (PAN) ${ }^{(18)}$.

Los CAP se suscribieron entre el MEF con los GR de Apurímac, Ayacucho y Huancavelica por ser las regiones con las mayores prevalencias de DCl en 2008 (34\% en

\section{MENSAJES CLAVE}

Motivación para realizar el estudio. La evidencia sobre los efectos de esquemas de incentivos en salud es mixta y poco concluyente. A partir de esta evaluación se busca generar información de desempeño que sustenten mejoras en su diseño.

Principales hallazgos. El esquema habría sido efectivo en activar la cadena mayor cobertura de vacunas completas y de rotavirus, menor incidencia de EDA y DCI, pero no mejoró la cobertura de otras prestaciones como CRED y entrega de suplementos de hierro al niño y gestante, donde la interacción entre el personal de salud y el paciente es crítica para aumentar la demanda del servicio. Los hallazgos también alertan sobre la falta de sostenibilidad del mecanismo.

Implicancias. Hay un margen de mejora en el diseño del esquema para potenciar resultados.

Ayacucho, 37\% en Apurímac y 52\% en Huancavelica). A través de los CAP, el Gobierno nacional asignó transferencias de recursos adicionales al presupuesto institucional de dichas regiones en función del cumplimiento de metas y compromisos consensuados y formalizados en los convenios suscritos, las que priorizaron las siguientes prestaciones de salud orientadas a reducir la $\mathrm{DCl}$, según el marco lógico del PAN ${ }^{(19)}$ y que se entregan en los establecimientos de salud públicos de primer nivel de atención: aplicación de vacunas básicas completas a menores de 5 años, atenciones para el control de crecimiento y desarrollo (CRED) del niño, suplementación de hierro y vitamina A a menores de 3 años y de hierro y ácido fólico a madres gestantes y puérperas, según esquema y normas técnicas vigentes establecidas por el Ministerio de Salud (20).

EI CAP estaba compuesto por dos tramos: uno "variable", que pagaba de acuerdo al cumplimiento de metas en los indicadores de coberturas delos servicios desalud priorizados y uno "fijo", que pagaba de acuerdo al cumplimiento de compromisos de gestión orientados a mejorar los procesos operativos de la oferta de las prestaciones en los centros de salud. De esta manera, la cadena de resultados del CAP incentivaría un mayor esfuerzo de los establecimientos de salud públicos por incrementar los indicadores de cobertura de las prestaciones priorizadas $y$, a través de dichos incrementos, se mejoraría el estado nutricional de los niños menores de 5 años en sus jurisdicciones, medido a través de la menor incidencia de enfermedades prevalentes causantes de la $\mathrm{DCl}$ (diarreas e infecciones respiratorias) y a través de la reducción de la $\mathrm{DCl}$ y anemia ${ }^{(19,20)}$.

La duración de los CAP fue cuatro años desde la fecha de suscripción (junio de 2010). El ámbito territorial en el que se evaluaría el cumplimiento de las metas sería los 54 distritos ubicados en el quintil más pobre de las tres regiones beneficiarias en conjunto, aunque no se impuso ninguna restricción geográfica en la ejecución de 
los fondos. En la Tabla 1 se reportan los desembolsos máximos anuales, según el nivel de cumplimiento de las metas y compromisos de gestión (respecto al año anterior), los que podían representar hasta el $30 \%$ del presupuesto del GR en el PAN.

Al finalizar los CAP, el Gobierno continuó implementando esquemas similares para alinear incentivos de los trabajadores de salud a los objetivos de salud pública y para otros objetivos de política nacional, pese a la falta de evidencia del impacto de este mecanismo. Asimismo, diversas revisiones de evidencia advierten sobre el poco consenso sobre la efectivdad de diferentes esquemas de pagos por desempeño aplicados por Gobiernos para logros en salud ${ }^{(14,21,22)}$.

Precisamente por ello, este estudio busca estimar el impacto promedio atribuible a la suscripción de los CAP sobre los indicadores que miden la cobertura de los servicios de salud priorizados y el estado nutricional de los niños menores de 5 años en las zonas en donde fue implementado entre 2010 y 2014 . Es decir, medir la eficacia del mecanismo subyacente a los CAP para potenciar los resultados de la política de reducción de la desnutrición infantil. Este objetivo resulta relevante porque generaría información de desempeño para sustentar mejoras en el diseño del mecanismo y orientar la política pública hacia intervenciones efectivas.

\section{MATERIALES Y MÉTODOS}

\section{ESTIMACIÓN DEL EFECTO PROMEDIO SOBRE LOS TRATADOS A PARTIR DEL MODELO DE DIFERENCIAS EN DIFERENCIAS}

Para estimar el impacto de los CAP se implementó un diseño de evaluación cuasiexperimental a partir de la comparación estadística de los indicadores de resultados entre una muestra de niños beneficiarios y una muestra de los niños de control, entre el periodo previo al inicio de los CAP (2008 y 2009) y diferentes mediciones posintervención, durante los años en que el mecanismo estuvo vigente (2010 a 2012) y cuando ya había finalizado (2013 y 2014), para analizar la evolución y sostenibilidad de los efectos en el tiempo. Ello permitió reportar los estimadores de diferencias en diferencias del impacto promedio de los convenios sobre los beneficiarios.

La población de estudio fueron niños de 0 a 59 meses $\mathrm{y}$, dependiendo del indicador, se restringió a niños de 0 a 36 meses y de 0 a 24 meses, o gestantes durante el periodo 2010-2014. Se seleccionaron los hogares con niños menores de 5 años y/o gestantes de una muestra de distritos de la Encuesta Nacional de Demografía y Salud Familiar (ENDES) entre 2008 a 2014.

\section{DEFINICIÓN DE ESCENARIOS}

Consideramos dos escenarios: en el escenario uno se tomó como grupo de tratamiento a los hogares de los distritos de las tres regiones donde se suscribieron los CAP, y como grupo control a los hogares de los distritos que se encontrarán fuera del ámbito de dichas regiones.

En el escenario dos, se seleccionaron dos subgrupos similares a los del escenario uno (regiones con CAP y regiones sin $\mathrm{CAP}$ ), y que, además, pertenecieran a distritos del primer quintil de pobreza.

Para seleccionar la muestra distrital, se buscó aquellos distritos de las regiones que suscribieron los CAP similares en sus condiciones iniciales a aquellos fuera del ámbito de los CAP, a través de la técnica del propensity score matching (PSM), para asegurar que la muestra esté balanceada en una serie de características socioeconómicas distritales que condicionarían la trayectoria de los resultados. Dichas características se tomaron del Censo de Población y Vivienda 2007 y de los mapas de pobreza y desnutrición (2007 y 2009). Estas fueron porcentaje de pobres y pobres extremos, DCl, población, ingreso per cápita, porcentaje de mujeres analfabetas, porcentaje de población rural, porcentaje de hogares con acceso a agua, número necesidades básicas insatisfechas.

En la Tabla 2 se definen los indicadores de resultado sobre los que se evaluó el impacto de los CAP según su teoría de cambio (para mayor detalle, véase Cruzado ${ }^{(23)}$ ). Además de los indicadores de coberturas de las prestaciones de salud priorizadas, se evaluó también el efecto en la reducción de la incidencia de EDA e IRA en menores de 5 años, y en el estado nutricional infantil ( $\mathrm{DCl}$ y anemia), recogidos de la ENDES.

Estimamos el efecto promedio del tratamiento sobre los tratados de los CAP (ATT, por sus siglas en ingles $=E\left[Y_{1 i}\right.$ $\left.\left.\left.Y_{0 i} \mid D=1\right]\right) ;\right) ;$ que representa el incremento promedio en los resultados de los niños/gestantes que residen en los distritos de las regiones donde se suscribieron los CAP a partir del estimador de diferencias en diferencias del ATT:

$$
\begin{aligned}
& \delta A T T=A T T_{C A P}^{(T=1)}+\left(E\left[Y_{0 i}^{(T=1)}-Y_{0 i}^{T=0} \mid D=1\right]\right)-\left(E\left[Y_{0 i}^{(T=1)}-Y_{0 i}^{T=0} \mid D=0\right]\right) \\
& \delta A T T=\left(E\left[Y_{1 i}^{(T=1)} \mid D=1\right]\right)-\left(E\left[Y_{0 i}^{T=0} \mid D=1\right]\right)-\left(E\left[Y_{1 i}^{T=1} \mid D=1\right]\right)-\left(E\left[Y_{0 i}^{T=0} \mid D=0\right]\right) \\
& D=1 \text { si el niño vive en un distrito de una región CAP y } 0 \text { en caso } \\
& \text { contrario. } \\
& T=1 \text { en los años de seguimiento, } T=0 \text { en los años de línea de base }
\end{aligned}
$$

Dicho estimador permite, a través de los efectos fijos distritales, eliminar sesgos debido a la heterogeneidad específica a cada distrito invariantes durante el periodo 
Tabla 1. Montos transferidos por cumplimiento de metas y compromisos de gestión*

\begin{tabular}{|c|c|c|c|c|c|c|c|c|c|c|c|c|c|c|c|c|}
\hline \multirow{2}{*}{ Región } & \multirow{2}{*}{ Tramo } & \multicolumn{5}{|c|}{ Montos máximos† } & \multicolumn{5}{|c|}{ Montos transferidos $†$} & \multicolumn{5}{|c|}{$\begin{array}{l}\text { Porcentaje de cumplimiento de } \\
\text { meta }\end{array}$} \\
\hline & & $\begin{array}{c}\text { Año } \\
1\end{array}$ & $\begin{array}{c}\text { Año } \\
2\end{array}$ & $\begin{array}{c}\text { Año } \\
3\end{array}$ & $\begin{array}{c}\text { Año } \\
4\end{array}$ & Total & $\begin{array}{c}\text { Año } \\
1\end{array}$ & $\begin{array}{c}\text { Año } \\
2\end{array}$ & $\begin{array}{c}\text { Año } \\
3\end{array}$ & $\begin{array}{c}\text { Año } \\
4\end{array}$ & Total & $\begin{array}{c}\text { Año } \\
1\end{array}$ & $\begin{array}{c}\text { Año } \\
2\end{array}$ & $\begin{array}{c}\text { Año } \\
3\end{array}$ & $\begin{array}{c}\text { Año } \\
4\end{array}$ & Total \\
\hline \multirow{3}{*}{ Apurímac } & Fijo & 12 & 8,9 & 6,4 & 3,8 & 31 & 7,2 & 5,3 & 3,8 & 2,3 & 18,6 & 60 & 60 & 60 & 60 & 60 \\
\hline & Variable & - & 3,8 & 6,4 & 8,8 & 19 & - & 1,2 & 3,6 & 7,7 & 12,4 & - & 30 & 56 & 88 & 66 \\
\hline & Total & 12 & 12,7 & 12,7 & 12,6 & 50 & 7,2 & 6,5 & 7,4 & 10 & 31,1 & 60 & 51 & 58 & 79 & 62 \\
\hline \multirow{3}{*}{ Ayacucho } & Fijo & 13,5 & 8,8 & 6 & 3,6 & 31,9 & 8,1 & 5,3 & 6 & 3,6 & 23 & 60 & 60 & 100 & 100 & 72 \\
\hline & Variable & - & 3,8 & 6 & 8,4 & 18,2 & - & 3,5 & 5,3 & 8,4 & 17,2 & - & 94 & 88 & 100 & 95 \\
\hline & Total & 13,5 & 12,5 & 12 & 12 & 50 & 8,1 & 8,8 & 11,3 & 12 & 40,1 & 60 & 70 & 94 & 100 & 80 \\
\hline \multirow{3}{*}{ Huancavelica } & Fijo & 12,5 & 8,8 & 6,3 & 3,8 & 31,3 & 7,5 & 5,3 & 6,3 & 3,8 & 22,8 & 60 & 60 & 100 & 100 & 73 \\
\hline & Variable & - & 3,8 & 6,3 & 8,8 & 18,8 & - & 3,8 & 5,9 & 8,2 & 17,8 & - & 100 & 94 & 94 & 95 \\
\hline & Total & 12,5 & 12,5 & 12,5 & 12,5 & 50 & 7,5 & 9 & 12,1 & 12 & 40,6 & 60 & 72 & 97 & 96 & 81 \\
\hline
\end{tabular}

* La evaluación del cumplimiento de metas y compromiso de gestión estuvo a cargo del Ministerio de Economía y Finanzas

† En millones de nuevos soles.

‡ Componentes del convenio de apoyo presupuestario; tramo fijo: pago condicionado a compromiso de gestión; tramo variable: condicionado a cumplimiento de metas

Años 1, 2, 3 y 4 corresponden al 2010, 2011, 2012 y 2013, respectivamente. Se evaluó el cumplimiento respecto al año anterior.

Fuente: Elaboración propia a partir de la Consulta Amigable del Sistema Integrado de Administración financiera

de análisis (características socioeconómicas, acceso a servicios básicos, oferta de salud, institucionalidad de las autoridades locales y regionales y factores climáticos que podrían afectar las coberturas de servicios de salud), y por shocks temporales comunes a todos los distritos.

Sin embargo, para que $\delta$ ATT represente un estimador no sesgado del ${ }_{A T T c a p}$ supuesto es que en ausencia de los CAP, la tendencia de los indicadores evaluados sería la misma en ambos grupos. Para evaluar su plausibilidad, realizamos pruebas placebo a partir de la estimación de regresiones de doble diferencias para una muestra de hogares de los distritos de tratamiento y control durante el periodo previo a los CAP (2008-2009). Bajo el supuesto de tendencias comunes, la hipótesis nula de que el coeficiente es igual a cero no debería rechazarse.

El estimador de doble diferencias para cada una de las variables de resultado se calculó empleando un modelo de probabilidad lineal a partir de la siguiente ecuación reducida:

$\mathrm{y}_{\text {idt }}=\alpha+\gamma_{1} \mathrm{~T}_{\mathrm{CAP}}+\delta_{\mathrm{d}}+\lambda(\mathrm{t})+\delta_{\mathrm{ATT}}\left(\mathrm{T}_{\mathrm{CAP}}{ }^{*} \mathrm{t}\right)+\beta_{1} \mathrm{X}_{\mathrm{it}}+\varepsilon_{\text {idt }}[\mathrm{pw}=\mathrm{FE}]$

Donde:

i: $\quad$ Niño o gestante

t: $\quad$ Tiempo, ( $=1$ para cada una de las mediciones de seguimiento y $=0$ para la medición basal).

$\mathrm{T}_{\text {CAP }}$ : Identificador de tratamiento (1 si el niño pertenece a un distrito de una región CAP o un distrito priorizado; $=0$ en caso contrario)

$\delta$ : $\quad$ Efectos fijos distritales

$\mathrm{y}_{\mathrm{ddt}}$ : Resultado en el año $\mathrm{t}$ del niño "i" que vive en el distrito "d".

FE: Factor de expansión obtenido del propense score matching

$\lambda$ : $\quad$ Parámetro que mide la evolución del indicador sobre el periodo de evaluación

$X_{\text {itt }} \quad$ Características socioeconómicas del niño: edad en meses y sexo del niño, edad y años de educación de la madre, si la madre usa televisión, radio o lee periódico al menos una vez por semana, sexo del jefe del hogar, si la madre está viva, riqueza del hogar (reportada en la ENDES), ámbito rural, acceso a servicios de agua, si el niño es beneficiario de JUNTOS y beneficiario del Sistema Integral de Salud. Además, se incluyó la tasa de crecimiento del PBI regional y el gasto promedio provincial por niño en las prestaciones de salud.
Para analizar si existen impactos diferenciados sobre cada uno de los resultados de interés según el nivel de gasto provincial, se consideran interacciones con las variables que identifican si el distrito se encuentra en una provincia del tercil superior del ranking de gasto per capita con las variables de tiempo (TCAP), de tratamiento ) y con la interacción tiempo y tratamiento (txTCAP).

\section{ASPECTOS ÉTICOS}

Todas las fuentes de información empleadas en el presente estudio son de naturaleza secundaria y de acceso público.

\section{RESULTADOS}

En promedio, la muestra de distritos de tratamiento y control en 2009 tenían una prevalencia de $\mathrm{DCl}$ de $39 \%$, pobreza de $68 \%, 22 \%$ de analfabetismo, $66 \%$ de población rural y $60 \%$ de hogares sin acceso a red pública de agua. Asimimimo, dependiendo del indicador evaluado, las estimaciones emplearon una muestra de hasta 6388 niños menores de 5 años.

Entre los años 2010 y 2014, la cobertura de vacunas básicas completas de los niños del grupo de control varió entre 60 y $67 \%$, la vacuna de rotavirus entre 37 y $52 \%$; mientras que el número de controles CRED completos para su edad pasó de 36 a $39 \%$, la cobertura de suplemento de hierro y multimicronutrientes en niños de 17 a $21 \%$ y de hierro y de ácido fólico en gestantes de 78 a $81 \%$. La incidencia de niños con EDA se mantuvo en $10 \%$ mientras que la $\mathrm{DCl}$ y anemia en menores de 36 meses se redujo de 35 a $32 \%$ y de 51 a $49 \%$ respectivamente. Para el 2010, las proporciones de servicios descritos fueron comparables con el grupo de tratamiento, según prueba de medias.

Respecto a la cobertura de prestaciones de salud (Tabla 3), bajo el escenario uno se encontró un impacto 
Tabla 2. Matriz de indicadores de resultado de la evaluación

\begin{tabular}{|c|c|}
\hline Indicador & Descripción \\
\hline $\begin{array}{l}\text { Porcentaje de niños con } \\
\text { sus vacunas contra el } \\
\text { rotavirus completas *, } \|\end{array}$ & $\begin{array}{l}\text {-La vacuna contra rotavirus ataca episodios de diarrea. En el menor de } 6 \text { meses, se aplica } \\
\text { en dos dosis en el } 2 .^{\circ} \text { y } 4 .^{\circ} \text { mes, de presentación monodosis, cada dosis de } 1,5 \mathrm{~mL} \text { por vía oral. } \\
\text {-Cuando la vacunación con rotavirus no se administró con oportunidad, el niño podrá iniciar la primera } \\
\text { dosis hasta los } 4 \text { meses con un intervalo mínimo de } 1 \text { mes para la aplicación de la segunda dosis. }\end{array}$ \\
\hline
\end{tabular}

Porcentaje de niños con su vacuna contra el neumococo completas *, II

Porcentaje de niños con sus vacunas básicas completas $\dagger, \|$

Porcentaje de niños con sus vacuna completas $\dagger$, $\|$

Porcentaje de niños con

Control de Crecimiento

y Desarrollo completo

(CRED) para su edad $\ddagger$

Porcentaje de niños con suplemento de hierro y vitamina $\mathrm{A} \S$

Porcentaje de gestantes con suplemento de hierro y ácido fólico

Porcentaje de niños que con infección respiratorias agudas (IRA) $\ddagger$

Porcentaje de niños con enfermedad diarréica aguda (EDA) $\ddagger$

Porcentaje de niños con desnutrición crónica infantil (OMS) $\ddagger$

score $Z$ del niño $\ddagger$ el niño, según una distribución de talla para la edad de referencia. Se utiliza la distribución de la Organización Mundial de la Salud como referencia.

Es una condición en la cual la sangre carece de suficientes glóbulos rojos o la concentración de Porcentaje de niños con anemia §

-La vacuna contra el neumococo ataca infecciones respiratorias. En los niños hasta los 12 meses, se aplican tres dosis: al $2 .^{\circ}$ mes, $4 .^{\circ}$ mes y 12 meses. Se aplica $0,5 \mathrm{~mL}$, porvía intramuscular en el tercio medio de la cara anterolateral externa del muslo, con jeringa descartable y aguja retráctil de $1 \mathrm{~mL}$ y aguja $25 \mathrm{G} \times 1$ ". -En los niños entre 12 y 23 meses y 29 días no vacunados previamente dos dosis con intervalo de al menos 1 mes entre dosis.

Niño menor de 3 años que ha recibido todas las vacunas del Esquema Nacional de Vacunación según cronograma estipulado para su edad. Comprende: una dosis de BCG (contra la tuberculosis), tres dosis de DPT (contra la difteria, pertussis o tos ferina y tétano) y 1 dosis contra el sarampión.

Es el porcentaje de vacunas básicas completas del niño menor de 36 meses. Incluye: 1 dosis de BCG, 3 dosis de DPT/Pentavalente, tres dosis contra la poliomielitis (2 IPV/1 APO) ,1 dosis contra el sarampión, 1 dosis antiamarílica y 1 dosis refuerzo DPT.

Atenciones periódicas donde se monitorea el crecimiento del niño. El cálculo del indicador considera que un niño tiene controles de crecimiento y desarrollo de acuerdo a su edad si cumple lo siguiente: Recién nacido al 7 y 15 día de vida

-Niños de 01 a 11 meses 01 control por mes.

-Niños de 12 a 23 meses 01 control cada 2 meses.

-Niños de 24 a 59 meses 01 control cada 3 meses.

Es aquel niño de 6 a 36 meses que ha recibido suplemento de hierro según esquema vigente. Implica 01 atención mensual por 12 meses e incluye indicación y entrega de insumo (Micronutrientes); consejería a la madre para promover la adherencia, monitoreo del consumo de los insumos a partir de la segunda atención. Adicionalmente, de acuerdo al esquema vigente se realizará el dosaje de hemoglobina de control.

La suplementación es una intervención que tiene como objetivo asegurar el suministro adecuado a las gestantes y puérperas de hierro más ácido fólico, para disminuir la prevalencia de anemia y otras complicaciones. Se entrega a partir de las 14 semanas de gestación cada vez que la usuaria acude a la consulta o en domicilio (180 tabletas de sulfato ferroso durante la gestación y 30 durante el puerperio).

Las infecciones respiratorias agudas son un complejo y heterogéneo grupo de infecciones causadas por distintos gérmenes, que afectan cualquier parte del aparato respiratorio. Se considera un caso de IRA si el niño menor de 36 meses presentó sintomas de infecciones respiratorias agudas en las últimas dos semanas.

Se define como diarrea a la presencia de tres o más deposiciones sueltas o líquidas en un periodo de 24 horas. Si un episodio de diarrea dura menos de 14 días, se trata de diarrea aguda. Si dura 14 días o más, se la denomina persistente, que es la que suele causar problemas nutricionales y puede producir la muerte de los niños y niñas con diarrea.

Es el niño menor de 5 años cuya talla para la edad está dos desviaciones estándar por debajo de la mediana del patrón de crecimiento internacional tomado como población de referencia (patrón OMS).

hemoglobina es menor que los valores de referencia según edad sexo y altitud. Se considera que el niño con edad entre 6 y menos de 36 meses tiene anemia por déficit de hierro si tienen menos de $11 \mathrm{~g}$ de hemoglobina por decilitro de sangre.

* El indicador se calculó para niños menores de 24 meses y para niños menores de 5 años

† El indicador se calculó para niños menores de 36 meses y para niños menores de 5 años

§ El indicador se calculó para niños entre 6 y 36 meses

I| De acuerdo a la Resolución Ministerial 651-2016/MINSA, que aprueba la NTS 080-MINSA/DGIESP V.04, "Norma Técnica de Salud que establece el Esquema Nacional de Vacunación"

Fuente: Elaboración propia a partir de anexo 2 del PAN, Ministerio de Salud 
significativo de 15 puntos porcentuales ( $p p$, en adelante) en la probabilidad de que el niño menor de 5 años tenga sus vacunas básicas completas solo durante el primer año de implementación. Sin embargo, sí se encotraron incrementos de 9, 14 y 12 pp (en la especificación 4) en la probabilidad de que el niño menor de 5 años tenga sus vacunas completas durante los 3 años de implementación de los CAP. Los mismos indicadores para niños menores de 3 años no mostraron impactos positivos significativos.

Bajo el escenario 2 (Tabla 4), se encontraron impactos de mayor valor en ambos indicadores. Así, por ejemplo, reportamos incrementos de $34 \mathrm{pp}$ en la probabilidad de vacunas básicas completas y de hasta 42 y 40 pp en la probabilidad de vacunas básicas completas en niños menores de 5 años, durante el periodo de vigencia del CAP. Aunque se encontraron algunos impactos significativos en menores de 3 años, las estimaciones fueron menos precisas pues mostraban desviaciones estándar altas.

No se encontraron los efectos positivos esperados en la proporción de niños con sus CRED completos para su edad en ningún periodo y en ninguno de los escenarios analizados. Incluso, se observaron algunos efectos negativos mientras los CAP estuvieron vigentes.

Salvo la especificación 2 en menores de 5 años, no se encontraron impactos positivos robustos en la proporción de niños con sus vacunas contra el neumococo en ninguno de los escenarios, mientras que en el caso del rotavirus, en cambio, sí hubo incrementos significativos entre 9 y 12 pp durante los dos primeros años de vigencia de los CAP para menores de 5 años y de 16 a 19 para menores de 3 años en el primer año, bajo el escenario 1. El escenario 2 reportó impactos positivos durante el primer año y después del término del programa en menores de 5 años, pero no en menores de 3.

En general, los efectos hallados sobre vacunas se redujeron a partir del tercer año de implementación y se anularon en los años inmediatamente posteriores al CAP. Asimismo, a excepción del caso de vacunas básicas completas para menores de 3 años, las pruebas placebo relativas a estos indicadores no mostraron impactos significativos en el periodo previo a la implementación (véase columna 5 de la Tabla 3 )

Se encontró un incremento significativo en la proporción de niños entre 6 y 36 meses que recibieron suplemento de hierro sólo durante el primer año de implementación del CAP (entre 10 y 14 pp en menores de 5 años) y entre 11 y 16 pp en menores de 3 años, bajo el escenario 1. Para el resto de años, los efectos fueron no significativos e incluso negativos, contrario a lo esperado. El escenario 2 reportó impactos negativos a partir del segundo año de vigencia del programa (para menores de 5 y 3 años). Se encontró también impactos negativos, contrariamente a lo esperado, en la probabilidad de gestantes con suplemento de hierro durante la vigencia de los CAP, bajo ambos escenarios.

Respecto al estado de salud del menor, bajo el escenario 1 (Tabla 5) se encontró un impacto de 8 pp en la reducción de la incidencia de EDA en el segundo año de implementación en menores de 5 y de 12 pp en menores de 3 años, coherente con los efectos encontrados en la cobertura de la vacuna de rotavirus. Este efecto no se sostuvo bajo el escenario 2 (Tabla 6).

El incremento en la cobertura de vacunas y la reducción en la proporción de niños con EDA atribuible a los CAP parecen reflejarse en la reducción de la $\mathrm{DCl}$ en los años posteriores al CAP, entre 10 pp en menores de 5 años y entre 13 y 17 pp en menores de 3 , bajo el escenario 1 . Este efecto es consistente con el incremento en el z score promedio de 17 pp para menores de 5 y entre 22 y 25 pp para menores de 3 en el mismo periodo. El escenario 2 reportó reducciones mayores en la $\mathrm{DCl}$ en el periodo posterior a los CAP, entre 37 y 46 pp en menores de 5 y entre 32 y 42 pp en menores de 3 años. Sin embargo, no se encontaron los impactos esperados en la anemia durante la vigencia de los CAP, los que muestran signos positivos en algunos casos. Las estimaciones placebo no reportaron efectos significativos.

\section{DISCUSIÓN}

Los resultados indicarían un efecto significativo y robusto atribuible a los CAP en la reducción de la $\mathrm{DCl}$ al final del periodo, a través de la reducción de la incidencia de EDA y del incremento de cobertura de todas las vacunas completas y de la vacuna de rotavirus durante los primeros años de vigencia del CAP.

Si bien el canal vinculado a la mayor cobertura de vacunas de rotavirus, menor incidencia de EDA y menor prevalencia de desnutrición pareciera haber funcionado, no se encontraron los efectos esperados y robustos en el resto de indicadores sobre los que se definieron las metas específicas del tramo variable: proporción de niños con sus controles de crecimiento y desarrollo, proporción de niños que recibieron suplementos de hierro, y proporción de madres gestantes con suplemento de hierro. Estos resultados no implican que las coberturas de dichos indicadores cayeran en las regiones beneficiarias de los CAP, pues el cumplimiento de las metas fue alto, sino que las coberturas se incrementaron aproximadamente en la misma magnitud (o mayor) en las regiones de control. Consistente con este patrón, los efectos sobre la anemia en menores de 3 años mostraron una dirección contraria a la esperada. 
Tabla 3. Impactos estimados en coberturas de servicios de salud en el escenario uno.

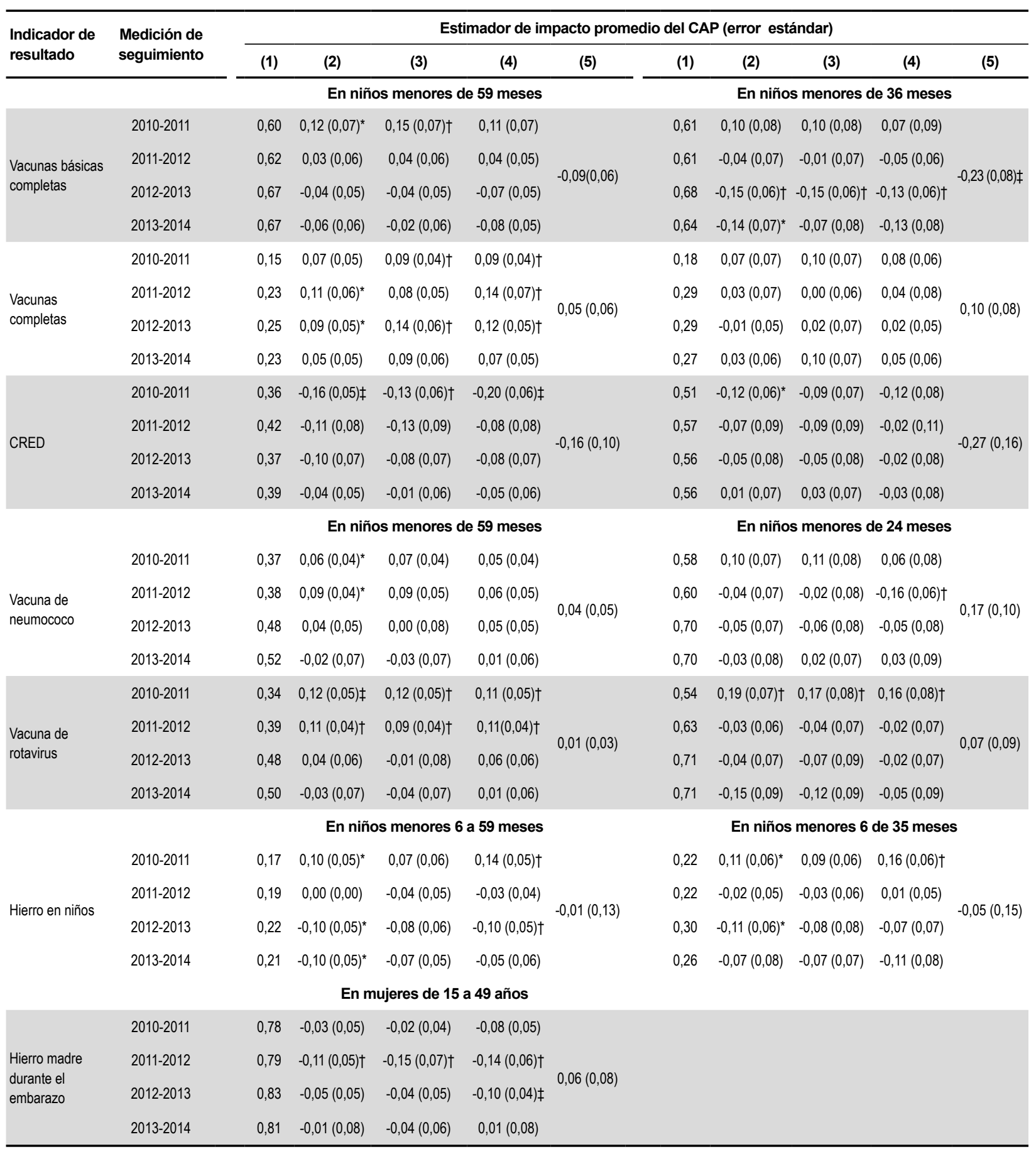

(1) Proporción del indicador en el grupo de comparación (2) Estimación de doble diferencia con efectos fijos regionales (3) Estimación de doble diferencia con gasto provincial (4) Estimación de doble diferencia con efectos fijos distritales (5) Estimación de doble diferencia para el período previo a la implementación de los CAP (2008-2009) para verificar cumplimiento de supuestos de tendencias comunes.

En todos los indicadores, a excepción del indicador vacunas básicas completas en niños menores de 3 años, el estimador de diferencias en diferencias no es significativo, lo que apuntaría al cumplimiento del supuesto.

El valor reportado en las columna (3) de la primera fila de la tabla 3 se interpreta de la siguiente manera: la probabilidad de los niños que residen en regiones donde se suscribió el CAP, para tener sus vacunas básicas completas es 15 puntos porcentuales más alta comparado con aquellos que residen en regiones donde no se suscribió el CAP.

CRED: control de crecimiento y desarrollo; CAP: convenio de apoyo presupuestario $\ddagger p<0,01, \uparrow p<0,05,{ }^{*} p<0,1$ 
Tabla 4. Impacto estimados en coberturas de servicios de salud en el escenario dos.

\begin{tabular}{|c|c|c|c|c|c|c|c|c|c|}
\hline \multirow{2}{*}{$\begin{array}{l}\text { Indicador } \\
\text { de } \\
\text { resultado }\end{array}$} & \multirow{2}{*}{$\begin{array}{l}\text { Medición de } \\
\text { seguimiento }\end{array}$} & \multicolumn{8}{|c|}{ Estimador de impacto promedio del CAP (error estándar) } \\
\hline & & (1) & (2) & (3) & (4) & (1) & (2) & (3) & (4) \\
\hline & & \multicolumn{4}{|c|}{ En niños menores de 59 meses } & \multicolumn{4}{|c|}{ En niños menores de 35 meses } \\
\hline \multirow{4}{*}{$\begin{array}{l}\text { Vacunas } \\
\text { básicas } \\
\text { completas }\end{array}$} & $2010-2011$ & 0,17 & $-0,16(0,24)$ & $0,03(0,21)$ & $-0,21(0,29)$ & 0,22 & $0,14(0,69)$ & $0,07(0,45)$ & $-0,12(0,90)$ \\
\hline & 2011-2012 & 0,17 & $0,39(0,11) \ddagger$ & $0,41(0,17) \dagger$ & $0,34(0,08) \ddagger$ & 0,18 & $0,50(0,21) \dagger$ & $0,42(0,24)$ & $0,59(0,20) \dagger$ \\
\hline & $2012-2013$ & 0,19 & $0,18(0,16)$ & $0,07(0,25)$ & $0,12(0,17)$ & 0,21 & $-0,07(0,37)$ & $-0,18(0,39)$ & $0,10(0,36)$ \\
\hline & 2013-2014 & 0,16 & $-0,08(0,06)$ & $-0,05(0,16)$ & $-0,09(0,06)$ & 0,18 & $-0,21(0,10)^{*}$ & $-0,08(0,24)$ & $-0,10(0,12)$ \\
\hline \multirow{4}{*}{$\begin{array}{l}\text { Vacunas } \\
\text { completas }\end{array}$} & $2010-2011$ & 0,63 & $0,36(0,18)^{\star}$ & $0,20(0,08) \dagger$ & $0,42(0,19)^{\star}$ & 0,64 & $-0,15(0,32)$ & $-0,06(0,27)$ & $0,06(0,59)$ \\
\hline & $2011-2012$ & 0,56 & $0,39(0,10) \mp$ & $0,35(0,13) \dagger$ & $0,40(0,07) \ddagger$ & 0,57 & $0,41(0,13) \dagger$ & $0,29(0,14)^{*}$ & $0,53(0,16) \dagger$ \\
\hline & $2012-2013$ & 0,62 & $0,21(0,08) \dagger$ & $0,25(0,11) \dagger$ & $0,15(0,10)$ & 0,61 & $0,05(0,14)$ & $0,03(0,14)$ & $0,12(0,17)$ \\
\hline & 2013-2014 & 0,64 & $0,24(0,09) \dagger$ & $0,39(0,11) \ddagger$ & $0,24(0,12)^{*}$ & 0,61 & $0,14(0,12)$ & $0,25(0,16)$ & $0,17(0,12)$ \\
\hline \multirow{4}{*}{ CRED } & 2010-2011 & 0,36 & $0,07(0,08)$ & $0,05(0,11)$ & $0,16(0,17)$ & 0,50 & $2,09(0,71)$ & $0,67(0,41)$ & $1,89(0,79)$ \\
\hline & 2011-2012 & 0,36 & $-0,24(0,15)$ & $-0,23(0,12)^{*}$ & $-0,28(0,11) \dagger$ & 0,50 & $-0,43(0,29)$ & $-0,38(0,19)^{*}$ & $-0,45(0,26)$ \\
\hline & 2012-2013 & 0,36 & $-0,17(0,13)$ & $-0,02(0,15)$ & $-0,15(0,15)$ & 0,50 & $-0,44(0,21)^{*}$ & $-0,24(0,28)$ & $-0,49(0,27)^{\star}$ \\
\hline & 2013-2014 & 0,40 & $-0,45(0,11) \ddagger$ & $-0,39(0,24)$ & $-0,47(0,15) \ddagger$ & 0,55 & $-0,45(0,14) \ddagger$ & $-0,53(0,22) \dagger$ & $-0,58(0,18) \ddagger$ \\
\hline & & \multicolumn{4}{|c|}{ En niños menores de 59 meses } & \multicolumn{4}{|c|}{ En niños menores de 35 meses } \\
\hline \multirow{4}{*}{$\begin{array}{l}\text { Vacuna de } \\
\text { neumococo }\end{array}$} & $2010-2011$ & 0,38 & $0,10(0,06)$ & $0,05(0,02) \dagger$ & $0,18(0,12)$ & 0,66 & $0,17(0,70)$ & $0,45(0,38)$ & $-0,35(0,65)$ \\
\hline & $2011-2012$ & 0,37 & $-0,20(0,08) \dagger$ & $-0,01(0,08)$ & $-0,20(0,06) \dagger$ & 0,62 & $0,33(0,26)$ & $0,40(0,31)$ & $0,33(0,26)$ \\
\hline & $2012-2013$ & 0,48 & $-0,09(0,13)$ & $-0,07(0,17)$ & $0,00(0,08)$ & 0,64 & $0,32(0,27)$ & $0,24(0,38)$ & $0,34(0,30)$ \\
\hline & 2013-2014 & 0,55 & $-0,04(0,07)$ & $0,00(0,18)$ & $-0,08(0,06)$ & 0,69 & $-0,69(0,23) \dagger$ & $-0,46(0,22)^{\star}$ & $-0,69(0,32)^{*}$ \\
\hline \multirow{5}{*}{$\begin{array}{l}\text { Vacuna de } \\
\text { rotavirus }\end{array}$} & $2010-2011$ & 0,35 & $0,20(0.10)^{*}$ & $0,10(0,04)^{*}$ & $0,27(0,16)$ & 0,55 & $-0,57(0,23)$ & $0,13(0,39)$ & $-0,76(0,36)$ \\
\hline & 2011-2012 & 0,35 & $-0,16(0,05)^{*}$ & $0,01(0,06)$ & $-0,16(0,03)^{\star}$ & 0,57 & $0,20(0,26)$ & $0,31(0,23)$ & $0,20(0,27)$ \\
\hline & $2012-2013$ & 0,47 & $0,06(0,09)$ & $0,06(0,12)$ & $0,12(0,11)$ & 0,68 & $0,10(0,23)$ & $0,05(0,28)$ & $0,10(0,29)$ \\
\hline & 2013-2014 & 0,53 & $0,20(0,06) \dagger$ & $0,14(0,22)$ & $0,17(0,08)^{*}$ & 0,68 & $-0,48(0,11)$ & $-0,35(0,14)$ & $-0,46(0,22)^{*}$ \\
\hline & & \multicolumn{4}{|c|}{ En niños entre 6 a 59 meses } & \multicolumn{4}{|c|}{ En niños entre 6 a 35 meses } \\
\hline \multirow{5}{*}{$\begin{array}{l}\text { Hierro en } \\
\text { niños }\end{array}$} & $2010-2011$ & 0,20 & $-0,10(0,24)$ & $-0,22(0,22)$ & $-0,22(0,15)$ & 0,28 & $-0,35(0,84)$ & $-0,43(0,16) \dagger$ & $-0,14(1,19)$ \\
\hline & 2011-2012 & 0,27 & $0,00(0,00)$ & $-0,43(0,05) \ddagger$ & $-0,39(0,10) \ddagger$ & 0,37 & $-0.53(0,22) \dagger$ & $-0,70(0,14) \ddagger$ & $-0,65(0,17) \ddagger$ \\
\hline & $2012-2013$ & 0,23 & $-0,22(0,09) \dagger$ & $-0,23(0,17)$ & $-0,19(0,12)$ & 0,33 & $-0,36(0,18)^{\star}$ & $-0.48(0,20) \dagger$ & $-0,48(0,19) \dagger$ \\
\hline & 2013-2014 & 0,22 & $-0,13(0,10)$ & $-0,04(0,14)$ & $-0,01(0,14)$ & 0,33 & $-0,29(0,11) \dagger$ & $-0.23(0,12)^{*}$ & $-0,25(0,19)$ \\
\hline & \multicolumn{5}{|c|}{ En mujeres de 15 a 49 años } & & & & \\
\hline \multirow{4}{*}{$\begin{array}{l}\text { Hierro madre } \\
\text { durante el } \\
\text { embarazo }\end{array}$} & $2010-2011$ & 0,81 & $-0,04(0,23)$ & $0,02(0,21)$ & $-0,26(0,05) \ddagger$ & & & & \\
\hline & 2011-2012 & 0,76 & $0,13(0,13)$ & $0,31(0,15)$ & $-0,05(0,08)$ & & & & \\
\hline & $2012-2013$ & 0,78 & $0,21(0,11)$ & $0,18(0,17)$ & $0,03(0,09)$ & & & & \\
\hline & 2013-2014 & 0,80 & $-0,05(0,06)$ & $-0,03(0,09)$ & $-0,16(0,07) \dagger$ & & & & \\
\hline
\end{tabular}

(1) Proporción del indicador en el grupo de comparación (2) Estimación de doble diferencia con efectos fijos regionales (3) Estimación de doble diferencia con gasto provincial (4) Estimación de doble diferencia con efectos fijos distritales.

El valor reportado en las columna (4) de la segunda fila de la tabla 4 se interpreta de la siguiente manera: la probabilidad de los niños que residen en regiones donde se suscribió el CAP, para tener sus vacunas básicas completas es 34 puntos porcentuales más alta comparado con aquellos que residen en regiones donde no se suscribió el CAP.

CRED: control de crecimiento y desarrollo; CAP: convenio de apoyo presupuestario $\ddagger p<0,01, \dagger p<0,05,{ }^{*} p<0,1$ 
Tabla 5. Impacto estimados en estado nutricional del niño por rango de edad (hasta 35 meses y hasta 59 mesesen el escenario uno.

\begin{tabular}{|c|c|c|c|c|c|c|c|c|c|c|c|}
\hline \multirow{2}{*}{$\begin{array}{l}\text { Indicador } \\
\text { de } \\
\text { resultado }\end{array}$} & \multirow{2}{*}{$\begin{array}{l}\text { Medición de } \\
\text { seguimiento }\end{array}$} & \multicolumn{10}{|c|}{ Estimador de impacto promedio del CAP (error estándar) } \\
\hline & & (1) & (2) & (3) & (4) & (5) & (1) & (2) & (3) & (4) & (5) \\
\hline & & \multicolumn{5}{|c|}{ En niños menores de 59 meses } & \multicolumn{5}{|c|}{ En niños menores de 36 meses } \\
\hline \multirow{4}{*}{$\begin{array}{l}\mathrm{DCl} \\
\text { según } \\
\text { criterio } \\
\text { OMS }\end{array}$} & $2010-2011$ & 0,35 & $-0,075(0,07)$ & $-0,08(0,07)$ & $-0,03(0,07)$ & \multirow{4}{*}{$-0,09(0,10)$} & 0,35 & $-0,17(0,09)^{*}$ & $-0,17(0,09)^{*}$ & $-0,11(0,11)$ & \multirow{4}{*}{$-0,11(0,13)$} \\
\hline & $2011-2012$ & 0,32 & $0,05(0,06)$ & $0,07(0,06)$ & $0,06(0,05)$ & & 0,30 & $0,08(0,07)$ & $0,09(0,08)$ & $0,11(0,07)$ & \\
\hline & 2012-2013 & 0,33 & $0,01(0,05)$ & $-0,03(0,05)$ & $-0,01(0,04)$ & & 0,32 & $-0,07(0,07)$ & $-0,02(0,06)$ & $0,05(0,07)$ & \\
\hline & 2013-2014 & 0,32 & $-0,10(0,05) \dagger$ & $-0,07(0,04)$ & $-0,10(0,04) \dagger$ & & 0,33 & $-0,13(0,06) \dagger$ & $-0,10(0,06)$ & $-0,17(0,06) \ddagger$ & \\
\hline \multirow{4}{*}{$\begin{array}{l}\mathrm{DCl} \\
\text { según } \\
\text { score Z }\end{array}$} & 2010-2011 & $-158,1$ & $16,63(16,22)$ & $21,02(17,20)$ & $12,63(17,43)$ & \multirow{4}{*}{$19,24(22,12)$} & $-152,47$ & $31,25(22,77)$ & $33,63(23,10)$ & $30,87(26,36)$ & \multirow{4}{*}{$0,99(28,40)$} \\
\hline & $2011-2012$ & $-150,22$ & $-29,12(11,31)$ & $-36,34(16,05)^{\star}$ & $-25,58(10,55)^{*}$ & & $-140,76$ & $-36,18(15,55)^{*}$ & $-40,90(20,53)^{*}$ & $-30,48(16,26)^{*}$ & \\
\hline & 2012-2013 & $-157,51$ & $-9,96(10,86)$ & $-1,92(11,69)$ & $-2,33(9,74)$ & & $-149,5$ & $7,37(15,34)$ & $13,78(16,46)$ & $-0,44(15,03)$ & \\
\hline & 2013-2014 & $-152,45$ & $14,12(9,94)$ & $8,94(10,37)$ & $17,52(9,99)^{*}$ & & $-149,31$ & $21,85(12,35)^{*}$ & $12,05(12,93)$ & $24,86(13,87)^{*}$ & \\
\hline \multirow{4}{*}{ IRA } & 2010-2011 & 0,11 & $0,02(0,05)$ & $0,02(0,04)$ & $0,05(0,04)$ & & 0,13 & $0,02(0,04)$ & $0,03(0,04)$ & $0,07(0,05)$ & \multirow{4}{*}{$-0,06(0,09)$} \\
\hline & 2011-2012 & 0,13 & $-0,04(0,05)$ & $-0,02(0,05)$ & $-0,02(0,05)$ & & 0,16 & $-0,01(0,06)$ & $0,01(0,06)$ & $0,03(0,07)$ & \\
\hline & 2012-2013 & 0,11 & $-0,00(0,05)$ & $0,02(0,05)$ & $0,03(0,04)$ & $-0,05(0,08)$ & 0,12 & $0,04(0,06)$ & $0,07(0,06)$ & $0,05(0,06)$ & \\
\hline & 2013-2014 & 0,15 & $0,092(0,05)^{*}$ & $0,10(0,04) \dagger$ & $0,07(0,05)$ & & 0,18 & $0,11(0,05) \dagger$ & $0,10(0,06)^{*}$ & $0,09(0,06)^{*}$ & \\
\hline \multirow{5}{*}{ EDA } & $2010-2011$ & 0,11 & $-0,05(0,05)$ & $-0,06(0,06)$ & $-0,03(0,04)$ & & 0,15 & $-0,06(0,07)$ & $-0,09(0,07)$ & $-0,03(0,06)$ & \multirow{5}{*}{$-0,14(0,08)$} \\
\hline & $2011-2012$ & 0,11 & $-0,07(0,05)$ & $-0,05(0,04)$ & $-0,08(0,04)^{*}$ & & 0,15 & $-0,12(0,06)^{*}$ & $-0,09(0,05)$ & $-0,12(0,05) \dagger$ & \\
\hline & & & & & & $-0,07(0,05)$ & & & & & \\
\hline & 2012-2013 & 0,09 & $0,04(0,04)$ & $0,04(0,04)$ & $0,01(0,04)$ & & 0,12 & $-0,01(0,05)$ & $0,00(0,05)$ & $-0,03(0,05)$ & \\
\hline & 2013-2014 & 0,10 & $0,10(0,05)$ & $0,08(0,05)$ & $0,04(0,05)$ & & 0,14 & $0,11(0,07)$ & $0,061(0,072)$ & $0,021(0,072)$ & \\
\hline
\end{tabular}

En niños entre 6 a 59 meses

\begin{tabular}{|c|c|c|c|c|c|c|c|c|c|c|c|}
\hline & 2010-2011 & 0,51 & $0,07(0,07)$ & $0,04(0,06)$ & $0,09(0,07)$ & & 0,60 & $0,16(0,09)^{*}$ & $0,13(0,08)$ & $0,20(0,10)^{*}$ & \multirow{5}{*}{$-0,17(0,17)$} \\
\hline \multirow{4}{*}{ Anemia } & 2011-2012 & 0,51 & $0,00(0,06)$ & $-0,03(0,07)$ & $0,00(0,06)$ & & 0,61 & $0,08(0,07)$ & $0,06(0,08)$ & $0,16(0,08)^{*}$ & \\
\hline & & & & & & $-0,08(0,11)$ & & & & & \\
\hline & 2012-2013 & 0,51 & $0,04(0,07)$ & $0,01(0,08)$ & $0,08(0,07)$ & & 0,61 & $0,18(0,08) \dagger$ & $0,15(0,09)^{*}$ & $0,27(0,08) \ddagger$ & \\
\hline & 2013-2014 & 0,49 & $0,03(0,07)$ & $-0,05(0,07)$ & $0,04(0,08)$ & & 0,61 & $0,00(0,08)$ & $-0,03(0,09)$ & $0,09(0,09)$ & \\
\hline
\end{tabular}

(1) Proporción del indicador en el grupo de comparación (2) Estimación de doble diferencia con efectos fijos regionales (3) Estimación de doble diferencia con gasto provincial (4) Estimación de doble diferencia con efectos fijos distritales (5) Estimación de doble diferencias para el periodo previo a la implementación de los CAP (2008-2009) para verificar cumplimiento de supuestos de tendencias comunes.

En todos los indicadores, el estimador de diferencias en diferencias no es significativo, lo que apuntaría al cumplimiento del supuesto.

CAP: convenio de apoyo presupuestario; DCl: desnutrición crónica infantil; OMS: Organización Mundial de la Salud; IRA: infección respiratoria aguda; EDA: enfermedad diarreica aguda. $\ddagger p<0,01, \dagger p<0,05,{ }^{*} p<0,1$ 
Tabla 6. Impactos estimados en estado nutricional del niño por rango de edad (hasta 35 meses y hasta 59 meses) en el escenario dos.

\begin{tabular}{|c|c|c|c|c|c|c|c|c|c|}
\hline \multirow{2}{*}{$\begin{array}{l}\text { Indicador de } \\
\text { resultado }\end{array}$} & \multirow{2}{*}{$\begin{array}{l}\text { Medición de } \\
\text { seguimiento }\end{array}$} & \multicolumn{8}{|c|}{ Estimador de impacto promedio del CAP } \\
\hline & & (1) & (2) & (3) & (4) & (1) & (2) & (3) & (4) \\
\hline & & \multicolumn{4}{|c|}{ En niños menores de 59 meses } & \multicolumn{4}{|c|}{ En niños menores de 36 meses } \\
\hline \multirow{4}{*}{$\begin{array}{l}\mathrm{DCl} \\
\text { según } \\
\text { criterio } \\
\text { OMS }\end{array}$} & 2010-2011 & 0,41 & $-0,18(0,22)$ & $0,00(0,10)$ & $-0,29(0,20)$ & 0,33 & $0,92(0,39)^{*}$ & $0,13(0,29)$ & $0,60(0,53)$ \\
\hline & 2011-2012 & 0,44 & $-0,04(0,23)$ & $-0,07(0,18)$ & $-0,19(0,16)$ & 0,40 & $0,04(0,28)$ & $-0,12(0,23)$ & $-0,10(0,26)$ \\
\hline & $2012-2013$ & 0,41 & $0,10(0,15)$ & $-0,11(0,19)$ & $-0,20(0,13)$ & 0,40 & $0,01(0,16)$ & $-0,14(0,18)$ & $0,06(0,23)$ \\
\hline & 2013-2014 & 0,42 & $-0,46(0,15) \dagger$ & $-0,41(0,17) \dagger$ & $-0,37(0,13) \dagger$ & 0,41 & $-0,32(0,14) \dagger$ & $-0, .41(0,19)^{*}$ & $-0,42(0,16) \dagger$ \\
\hline \multirow{4}{*}{$\begin{array}{l}\mathrm{DCl} \\
\text { según } \\
\text { score Z }\end{array}$} & 2010-2011 & $-179,01$ & $29,08(42,88)$ & $2,51(24,20)$ & $45,08(21,01)^{*}$ & $-159,58$ & $-130,73(60,68)^{*}$ & $-63,82(73,43)$ & $-120,87(140,49)$ \\
\hline & $2011-2012$ & $-185,03$ & $73,61(45,43)$ & $64,04(32,56)^{*}$ & $110,44(34,16) \dagger$ & $-172,86$ & $12,46(54,56)$ & $30,74(48,30)$ & $44,68(53,72)$ \\
\hline & $2012-2013$ & $-175,79$ & $18,78(28,69)$ & $40,07(42,95)$ & $93,36(31,90) \dagger$ & $-166,19$ & $11,26(51,43)$ & $47,09(49,78)$ & $0,47(50.88)$ \\
\hline & 2013-2014 & $-170,76$ & $56,71(24,69) \dagger$ & $51,31(30,03)$ & $44,05(25,50)$ & $-160,99$ & $26,64(28,61)$ & $50,73(44,37)$ & $25,80(36,19)$ \\
\hline \multirow{5}{*}{ IRA } & $2010-2011$ & 0,18 & $-0,31(0,18)$ & $-0,18(0,11)$ & $-0,03(0,09)$ & 0,19 & $-0,32(0,46)$ & $-0,52(0,18) \dagger$ & $-0,69(0,54)$ \\
\hline & 2011-2012 & 0,14 & $0,13(0,16)$ & $0,00(0,11)$ & $0,16(0,08)^{*}$ & 0,13 & $0,14(0,07)^{\star}$ & $0,09(0,05)$ & $0,20(0,08) \dagger$ \\
\hline & & & & & & & & & \\
\hline & 2012-2013 & 0,16 & $-0,08(0,09)$ & $0,00(0,14)$ & $0,07(0,07)$ & 0,17 & $0,05(0,10)$ & $0,15(0,17)$ & $0,02(0,10)$ \\
\hline & 2013-2014 & 0,15 & $0,08(0,06)$ & $0,14(0,08)$ & $0,15(0,07)^{*}$ & 0,17 & $0,45(0,08) \ddagger$ & $0,47(0,24)^{*}$ & $0,53(0,08) \ddagger$ \\
\hline \multirow{6}{*}{ EDA } & $2010-2011$ & 0,14 & $0,23(0,09)$ & $0,08(0,14)$ & $0,21(0,05)$ & 0,16 & $0,99(0,25)$ & $-0,08(0,26)$ & $0,94(0,21)$ \\
\hline & 2011-2012 & 0,14 & $0,00(0,08)$ & $0,03(0,08)$ & $0,01(0,05)$ & 0,15 & $0,39(0,12)$ & $0,34(0,14)$ & $0,48(0,10)$ \\
\hline & & & & & & & & & \\
\hline & $2012-2013$ & 0,12 & $-0,01(0,07)$ & $-0,04(0,08)$ & $0,08(0,07)$ & 0,15 & $0,11(0,17)$ & $0,00(0,17)$ & $0,30(0,11)$ \\
\hline & 2013-2014 & 0,10 & $0,10(0,07)$ & $0,00(0,09)$ & $0,05(0,06)$ & 0,14 & $0,14(0,10)$ & $-0,05(0,18)$ & $-0,05(0,09)$ \\
\hline & \multicolumn{5}{|c|}{ En niños entre 6 a 59 meses } & \multicolumn{4}{|c|}{ En niños entre 6 a 35 meses } \\
\hline \multirow{5}{*}{ Anemia } & 2010-2011 & 0,46 & $-0,06(0,17)$ & $-0,06(0,18)$ & $-0,10(0,16)$ & 0,58 & $-1,83(0,52) \dagger$ & $-0,07(0,51)$ & $-0,93(0,90)$ \\
\hline & $2011-2012$ & 0,54 & $-0,37(0,18)^{*}$ & $-0,32(0,16)^{*}$ & $-0,53(0,25)^{*}$ & 0,69 & $-0,93(0,23) \ddagger$ & $-0,73(0,33)^{*}$ & $-1,26(0,24) \ddagger$ \\
\hline & & & & & & & & & \\
\hline & $2012-2013$ & 0,51 & $-0,38(0,14) \dagger$ & $-0,21(0,15)$ & $-0,26(0,14)^{*}$ & 0,64 & $-0,65(0,21) \dagger$ & $-0,22(0,31)$ & $-0,78(0,18) \ddagger$ \\
\hline & 2013-2014 & 0,45 & $0,06(0,12)$ & $0,01(0,16)$ & $0,10(0,15)$ & 0,55 & $0,46(0,14) \ddagger$ & $0,33(0,27)$ & $0,61(0,23) \dagger$ \\
\hline
\end{tabular}

(1) Proporción del indicador en el grupo de comparación (2) Estimación de doble diferencia con efectos fijos regionales (3) Estimación de doble diferencia con gasto provincial (4) Estimación de doble diferencia con efectos fijos distritales.

CAP: convenio de apoyo presupuestario; DCl: desnutrición crónica infantil; OMS: Organización Mundial de la Salud; IRA: infección respiratoria aguda; EDA: enfermedad diarreica aguda ${ }^{* * *} p<0,01,{ }^{* *} p<0,05,{ }^{*} p<0,1$ 
Al respecto, la evidencia sobre los efectos de esquemas de incentivos en salud es mixta y poco concluyente. Miller y Singer ${ }^{(14)}$, a partir de una revisión de experiencias encontraron que los resultados de su aplicación no eran generalizables a todos los casos y que requerían un contexto apropiado. Por ejemplo, evaluaciones a pagos por desempeño a los establecimientos de salud y gobiernos subnacionales como el Plan Nacer de Argentina $^{(13)}$ encuentraron impactos significativos en el número de visitas de control prenatal y en la calidad de atención prenatal que redujeron el bajo peso al nacer y la mortalidad neonatal; mientras que en Ruanda (12), a diferencia de los CAP, se incrementó la probabilidad de que el niño complete sus visitas de control y en la probabilidad de recibir la vacuna antitétano durante la atención prenatal, pero no en la proporción de niños menores de 5 años con vacunas completas.

Las transferencias directas a los usuarios para incentivar la demanda de servicios salud encuentran efectos positivos en el cumplimiento de las corresponsabilidades vinculadas con la asistencia a los centros de salud. Sin embargo, en cuanto a la nutrición y estado de salud, los impactos son pequeños y sólo en ocasiones positivos (24-26). A diferencia del CAP, las evaluaciones de Juntos (27-29) encontraron impactos positivos en la demanda de servicios de salud pero no efectos claros sobre las tasas de desnutrición.

Por su parte Basani ${ }^{(20)}$ encuentró efectos modestos en la cobertura de vacunas, pero mayores en prácticas de lactancia y poco robustos en adopción de prácticas preventivas.

El diseño de los CAP no ha recogido metas de calidad o cumplimiento de protocolos en la prestación de los servicios, a pesar que una buena parte de la literatura señala que el pobre desempeño en los servicios de salud se debe a un déficit en el esfuerzo de los proveedores que interactúan con los usuarios, lo que ocasiona una baja calidad de la prestación médica o que el esfuerzo del proveedor podría no enfocarse directamente en mejorar la salud, sino en incrementos de cobertura (desincentivo). Incluso, aunque el proveedor de salud pueda ejecutar el esfuerzo requerido mientras dura la atención, pueden hacer poco por promover prácticas fuera de la consulta, según Eicher y Levine ${ }^{(30)}$.
Los resultados del estudio se reportan bajo diferentes especificaciones y muestras. La metodología aplicada permite controlar por variables no observables distritales invariantes en el tiempo y por condiciones iniciales que pueden haber determinado una trayectoria diferente entre los distritos beneficiarios. Las pruebas placebo apuntarían a la ausencia de sesgos no observables distritales. Sin embargo, algunas limitaciones están vinculadas a la posible presencia de sesgo no observables del niño pues no se dispone de data longitudinal, al limitado número de observaciones en los quintiles más pobres de las tres regiones que restan precisión en la identificación de beneficiarios, fórmula de cálculo de algunos indicadores de resultado y al uso de información secundaria.

Los hallazgos del presente estudio alertan sobre la falta de sostenibilidad del mecanismo de incentivos de los CAP, que podría deberse a un desgaste o a que las metas pudieron alcanzarse fácilmente, tal es así que las regiones sin convenio alcanzaron coberturas similares. Asimismo, la ausencia de efectos en la cobertura de los CRED y suplementos de hierro pudieran deberse al nivel de la meta o a que, a diferencia de las vacunas, donde la cadena de producción vinculada a la oferta resulta crítica para aumentar la cobertura, en las atenciones donde se controla el crecimiento y desarrollo del niño y en los controles prenatales, donde se entregan los suplementos de hierro del niño y a las gestantes, se requiere de una mejor interacción entre el personal de salud y el paciente para aumentar la demanda del servicio, y generar un cambio de conducta en los usuarios que repercuta en el estado nutricional del menor.

Agradecimientos: la autora agradece la valiosa colaboración de Juan José Cueto en los análisis realizados.

Contribuciones de autoría: la autora realizó la concepción y diseño del artículo, análisis e interpretación de los datos, redacción del borrador del artículo, revisión crítica y aprobación de la versión final.

Fuentes de financiamiento: Autofinanciado

Declaración de conflictos de interés: La autora declara no tener conflictos de interés.

\section{REFERENCIAS BIBLIOGRÁFICAS}

1. Eldridge C, PalmerN. Performance-based payment: some reflections on the discourse, evidence y unanswered questions. Health Policy Plan. 2009;24(3):160-6. doi: $10.1093 /$ heapol/czp002

2. Makinson J, editor. Incentives for change: rewarding performance in national govern- ment networs [Internet]. London, United Kingdom: Public Services Productivity Panel;2010 [citado el 03 de agosto de 2016] Disponible en: http://webarchive.nationalarchives.gov.uk/20130129110402/ http:/www.hm-treasury.gov.uk/media/601/A3/240.pdf
3. Corporación Andina de Fomento (CAF): Un estado más efectivo, Capacidades para el diseño, la implementación y el aprendizaje de políticas públicas [Internet]. Bogotá, Colombia: CAF; 2015 [citado el 10 de agosto de 2016] Disponible en: http://scioteca.caf.com/bitstream/hand- 
le/123456789/755/reporte_economia desarrollo caf 2015 estado politicas publicas.pdf? sequence $=1$ \&isAllowed $=\mathrm{y}$

4. Holmstrom B, Milgrom P. Multitask principal-agent analyses: Incentive contracts, asset ownership, and job design. Journal Law Econ Organ. 1991; 7(Spec issue):24-52.

5. Bernheim BD, Whinston MD. Common agency. Econometrica. 1986;54(4):92342. doi: $10.2307 / 1912844$

6. Behrman JR, Parker SW, Todd PE, Wolpin KI. Aligning learning incentives of students and teachers: results from a social experiment in Mexican high schools. J Polit Econ. 2015;123(2):325-64. doi: $10.1086 / 675910$

7. Glewwe P, Ilias N, Kremer M. Teacher incentives. Am Econ J-Appl Econ. 2010;2(3):205-27.

8. Lavy V. Performance pay y teachers' effort, productivity, y grading ethics. Am Econ Review. 2009;99(5):1979-2011.

9. Vegas E, editor. Incentives to improve teaching, lessons for Latin America [Internet]. Washington, D.C.: World Bank; 2005 [citado el 21 de setiembre de 2016] Disponible en: http:// siteresources.worldbank.org/INTINDIA/4371432-1194542398355/21543233/ Incentives_to_Improve_Teaching.pdf

10. Contreras D, Rau T. Tournament incentives for teachers: evidence from a scaled-up intervention in Chile. Econ Dev Cult Change. 2012;61(1): 219-46. doi: $10.1086 / 666955$

11. Banerjee A, Deaton A, Duflo E. Health, health care, and economic development: wealt, health, y health services in rural Rajasthan. Am Econ Rev. 2004;92(2):326330. doi: $10.1257 / 0002828041301902$

12. Gertler P, Vermeersch. Using performance incentives to improve health outcomes, Impact evaluation series $\mathrm{N}^{\circ}$ 60 [Internet]. Washington D.C.: World Bank; 2012 [citado el 21 de octubre de 2016] Disponible en: https://openknowledge.worldbank.org/bitstream/ handle/10986/9316/WPS6100.pd$\mathrm{f}$ ? sequence $=1$ \&is Allowed $=\mathrm{y}$

13. Gertler P, Giovagnoli P, Martinez s. Rewarding provider performance to enable a healthy start to life, evidence for Argentina's plan Nacer [Internet]. Washington D.C.: World Bank; 2010 [citado el 01 de noviembre de 2016] Disponible en: http://documents.worldbank.org/curated/en/910221468002421288/pdf/ WPS6884.pdf

14. Miller G, Singer K. Pay-for-performance incentives in low- and middle-income country health programs, working paper 18932. Cambridge, M.A.: The National Bureau of Economic Research; 2013 [citado el 16 de noviembre de 2016] Disponible en: http://www.nber.org/papers/ w18932.pdf

15. Jack W. Contracting for health services: an evaluation of recent reforms in Nicaragua. Health Policy Plan. 2003;18(2):195-204.

16. Miller G, Luo R, Zhang L, Sylvia S, Shi Y, Foo P, et al. Effectiveness of provider incentives for anaemia reduction in rural China: a cluster randomized trial. BMJ. 2012;345:9e4809. doi: 10.1136/ bmj.e4809

17. Singh P. Performance pay and information: reducing child malnutrition in urban slums [Internet]. Munich, Alemania: Munich Personal RePEc Archive; 2011 [citado el 16 de noviembre de 2016]. Disponible en: https://mpra.ub.uni-muenchen. de/29403/1/MPRA_paper_29403.pdf

18. Convenio de financiación entre la Comunidad Europea y la República del Perú (Convenio $\mathrm{N}^{\circ}$ DCIALA/2009/021-564) [Internet]. Lima, Peru: Ministerio de Economía y Finanzas; 2009 [citado el 17 de noviembre de 2016]. Disponible en: https://www.mef.gob.pe/ contenidos/presu_publ/ppr/europan/ Convenio_marco_de_Financiacion.pdf

19. Ministerio de Salud (MINSA). Programa presupuestal, Programa articulado nutricional contenidos mínimos (Directiva $\mathrm{N}^{\circ} 003-2015-\mathrm{EF} / 50.01$, Resolución directoral $\mathrm{N}^{\circ} 021-2015-\mathrm{EF} / 50.01$ ) [Internet]. Lima, Perú: MINSA; 2017 [citado el 15 de marzo de 2017] Disponible en: https://www.minsa.gob. pe/presupuestales2017/archivos_apelacion/anexo2/anexo2-ARTICULLADO\%20NUTRICIONAL.pdf

20. Convenio de apoyo presupuestario a los programas presupuestarios estratégicos entre el Ministerio de Economía y Finanzas, a través de la Dirección General del Presupuesto Público, y el Gobierno Regional del departamento de Ayacucho [Internet]. Lima, Peru: Ministerio de Economía y Finanzas; 2010 [citado el 17 de noviembre de 2016] Disponible en: https://www. mef.gob.pe/contenidos/presu_publ/ppr/ europan/regiones/convenio_MEF_ayacucho.pdf

21. Bassani D, Arora P, Wazny K, Gaffey M, Lenters L, Bhutta Z. Financial incentives and coverage of child health interventions: a systematic review and meta-analysis. BMC Public Health. 2013;13(Suppl 3):S30. doi: $10.1186 / 1471-2458-13-S 3-S 30$

22.. Witter S, Fretheim A, Kessy FL, Lindahl AK. Paying for performance to improve the delivery of health interventions in low- and middle-income countries. Cochrane Database Syst Rev. 2012;(2):CD007899. doi: 10.1002/14651858.CD007899.pub2

23. Cruzado V. Evaluación de impacto de un sistema de pagos por desempeño para mejorar la salud infantil: los convenios de apoyo presupuestario del programa articulado nutricional en Perú [tesis de licenciatura]. Lima: Facultad de Ciencias Sociales, Pontificia Universidad Católica del Perú; 2017.

24. Gertler P. Do conditional cash transfers improve child health? evidence from PROGRESA's control randomized experiment. Am Econ Rev. 2004;94(2):336-41.

25. Berhman J, Hoddinott J. Programme evaluation with unobserved heterogeneity and selective implementation: the Mexican PROGRESA impact on child nutrition. Oxford B Econ Stat. 2005;67(4):547-569. doi: 10.1111/j.1468-0084.2005.00131.x

26. Paxson C, Schady N. Does money matter? the effects of cash transfers on child health and development in rural Ecuador. Econ Dev Cult Change. 2010;59(1):187-229. doi: $10.1086 / 655458$

27. Perova E, Vakis R. Welfare impacts of the "Juntos" program in Peru: evidence from a non-experimental evaluation [Internet]. Lima, Perú: JUNTOS; 2009 [citado el 12 de agosto de 2016] Disponible en: http://www.juntos.gob.pe/modulos/ mod_legal/archivos/Evaluacion_CuasiExperimental1.pdf

28. Escobal J y Benites S. Algunos impactos del programa JUNTOS en el bienestar de los niños: Evidencia basada en el estudio Niños del Milenio. Boletín de políticas públicas sobre infancia. 2012;5:19 p.

29. Sánchez, A. y M. Jaramillo. Impacto del programa JUNTOS sobre la nutrición temprana. Estudios Económicos. 2012;(23);53-66.

30. Eichler R, Levine R. Performance incentives for global health: potential and pitfalls. 1er ed. Washington, D.C: CENTER FOR GLOBAL DEVELOPMENT; 2009

31. Loevinsohn B, Harding A. Buying results? Contracting for health service delivery in developing countries. Lancet. 2005;366(9486):676-81. doi: 10.1016/ S0140-6736(05)67140-1

Correspondencia: Viviana Cruzado de la Vega Dirección: Calle Tomás Ramsey 965, dpto. 203. Magdalena del Mar, Lima, Perú.

Teléfono: (+51) 986642272.

Correo electrónico: cruzado.viviana@gmail.com 